\title{
Interesting and impactful research: on phenomena, theory, and writing
}

\author{
Gerard J. Tellis ${ }^{1}$
}

Published online: 12 September 2016

(C) Academy of Marketing Science 2016

Most researchers assume that research is a quest for truth. So, the discovery of truth would automatically be impactful. However, research is impactful not if it is true, but if it refutes the assumption (belief) of its audience (Davis 1971). Refuting the assumption of the audience is also what makes it interesting. The truthfulness of a proposition is of limited duration. Every proposition or theory will ultimately be proved wrong as new research emerges and knowledge accumulates. What matters is merely whether the research runs counter to what is currently assumed as true. That's interesting. That's impactful. Let's consider some examples.

\section{Four examples}

One of the most interesting and impactful theories in the last 200 years is the theory of evolution. Why so? The reason is that it refuted the strong beliefs of millions of people around the world that extant species of life were created by one or more gods. Darwin postulated that all the variety of living things evolved from simpler life forms. This theory is still interesting and controversial because millions of people around the world still strongly believe in creation. Indeed, in recent decades, some such believers have tried to espouse a rival theory to evolution called creationism. They want to suggest that creation is not merely a belief system but a scientific theory like evolution. Non-believers in evolution repeatedly throw up a simple classic counterexample to

Gerard J. Tellis

tellis@usc.edu

1 University of Southern California, Marshall School of Business, Los Angeles, CA 90089-0808, USA evolution. How could humans have evolved from monkeys? Thus, evolution is perennially interesting and impactful to many, because about 157 years after its first formal propounding, it still seems to refute common beliefs and perceptions.

Evolution is enormously impactful. It provides the most parsimonious explanation for the death of now extinct species, the origin of new species, the variety of existing species, and their seemingly exquisite match to the environment. It has also enlightened paleontology, genetics, embryology, and other natural sciences.

Similarly, the theory of relativity is perennially interesting and impactful. It's interesting because even today its premises refute our common misperceptions about the world. We think that time is absolute and speed is relative. But the theory suggests that time is relative and the speed of light (in a vacuum) is absolute and independent of the source. Likewise, the impact of the theory has been enormous, enlightening our understanding of the universe, light, space travel, time, gravity, and many other issues.

These two examples are classic ones from the field of natural science. Numerous examples exist in the field of economics, management, and marketing. I will cite just two, market pioneering and mental accounting.

For decades, economists proposed the theory of first-mover advantage, which said that the first to enter a market had some enduring advantage over later entrants to the market. Marketing researchers further elaborated that to mean overwhelming advantage in terms of market share, success, and long-term market leadership. However, Golder and Tellis (1993) refuted this notion of pioneering advantage attributing it to survival bias, self-report bias, and weak measures of pioneers. With good measures free from biases, they found that pioneers mostly fail, have low long-term market share, and are rarely long-term leaders in their markets. Because the 
authors refuted a strong theory in economics, marketing, and management, their thesis was interesting, has earned high Google citations, and won the Odell Award for Long Term Contributions in the Journal of Marketing Research. The thesis continues to be interesting. Many economists, strategists, and lawyers who have not heard of or do not believe in Golder and Tellis $(1993)^{1}$ continue to propose pioneering advantage as a reason for success. Reporters continue to cite first moveradvantage whenever they describe the success of a market entrant, casually redefining the market or the order of entry to show the advantage. Students continue to offer first-mover advantage as a reason for firms that succeed or late mover disadvantages for others that fail. Here again, casual redefinition of order of entry or the market is used to fit the conclusion.

Likewise, Richard Thaler's theory of mental accounting is highly interesting and impactful. Economists typically believe that a dollar is a dollar in every context. Likewise, investors believe that money should be moved among accounts to the one where returns are the maximum. Such beliefs rely on the fungibility of money. However, Thaler showed that consumers do not behave according to this principle. Rather they maintain separate mental accounts that violate this principle. A simple example is a consumer who saves in a bank account at very low interest a sum of money to purchase a home. The same consumer also maintains credit card debt of a lesser amount, at very high interest. The consumer can pay off all of his or her debt with some of the bank savings to reduce monthly interest payments - but will not. Thaler went on to propose his theory of mental accounting that explains a number of anomalies in people's borrowing, saving, and use of money. His work on mental accounting has led to thousands of citations and may yet earn him a Nobel prize. Thaler's work is perennially interesting, because it still is difficult for consumers to understand the fungibility of money and use it accordingly.

These four examples show how propositions that refute beliefs are inherently interesting and impactful.

\section{Characteristics of interesting}

Editors and reviewers use a variety of synonyms for interesting, like aha, wow, new, novel, punchline, surprising, counterintuitive, refutational, fascinating, insightful. Editors and reviewers may not go beyond these words to explain what they really want. This essay suggests that what is common to all these words is a premise that refutes the assumptions of the audience. What editors and reviewers really want is a premise that refutes what is assumed or believed. Before writing or presenting, the researcher can ascertain this fact by three

\footnotetext{
${ }^{1}$ Replications of Golder and Tellis have been very sparse.
}

simple questions: What have I found? What do people believe about it? Does the finding refute their belief? A positive answer justifies moving to the next step. A negative answer suggests further research. Many times authors fail to emphasize the interesting when they submit their papers. A few times they fail to do so even after getting the paper accepted.

Counter-intuitive premises vary in the degree to which they are interesting. This can be seen in a simple plot of audience response (vertical axis) to the refutational strength of a premise (horizontal axis) (see Fig. 1). Attention (red line) increases linearly with the strength of the refutation. However, persuasion or buy-in (yellow curve) is bell-shaped. Initially, the stronger the refutation, the greater the degree of buy in, up to a point (fascination). Beyond this point, the refutation earns more negative than positive response, leading to shock and ultimate rejection (absurd). So the ideal is to get a response of "interesting" or "fascinating" but not "absurd." For example, today evidence that the earth is round is overwhelming. So, a paper by members of the flat-earth society arguing why the earth is flat would be desk-rejected as absurd. Authors need to carefully craft their propositions to elicit the most positive buy-in from the widest audience without the paper being rejected as either boring or absurd.

Audiences themselves differ in their priors and thus in what they consider interesting. Take the example of evolution. Staunch believers of the theory would find a claim of evolution boring. Staunch believers of creationism would find a claim of evolution absurd. It's probably a majority in the middle who find it interesting. So, authors need to know their audiences and position and craft the proposition carefully to persuade the target audience.

\section{Where to begin?}

Every beginner faces this question. The classic answer that scholars give a beginner is start with the literature. Why so? The reasons are straightforward. The literature contains what is known. It also contains proven models and methods to guide the new researcher. Most importantly, the literature reveals limitations that the new researcher can address or gaps that the new researcher can fill. Others recommend that theories are useful as they enlighten data and enable one to see phenomena. But is this really true?

I would like to offer an alternative approach. The literature contains established theories, which make a large number of assumptions. By the time the novice has amassed the tools to understand these theories, he or she may have already become a believer of the theory's assumptions. Such beliefs may color the young researcher's perception of the world. He or she may 
Fig. 1 Audience reponse to refutation. Adapted from Tellis (2016), Effective advertising, 2nd edition, forthcoming

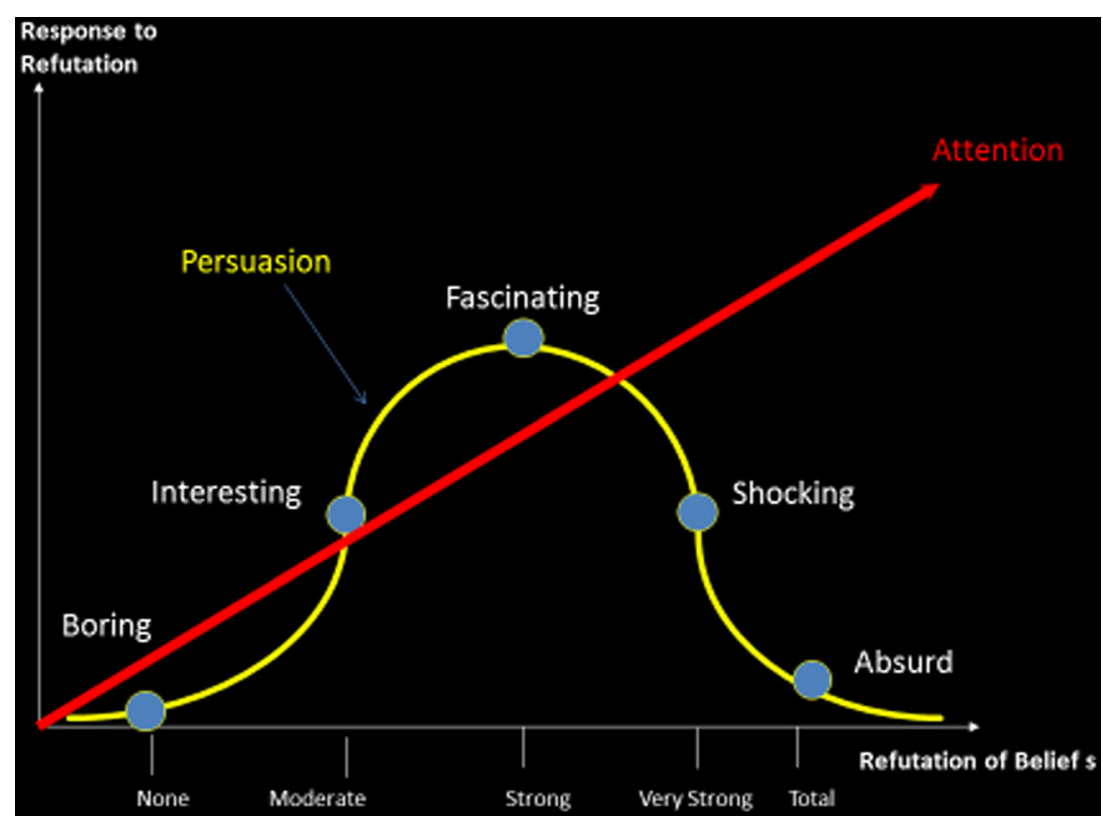

see the world through the eyes of the established researcher and be less likely to refute it. That may be why for generations, no economist challenged the belief in the fungibility of money, which Thaler finally refuted, or in pioneering advantage, which Golder and Tellis refuted.

Likewise, the literature contains methods and models that require training to understand. By the time the novice has fully understood these, his or her perception of the world and of data may be biased. Such biases may limit the novice's discovery of new phenomena. The list of limitations available at the end of existing literature could inspire the novice. However, the long list of limitations may also demotivate and discourage the novice into further research into the area.

So where to begin? Perhaps, begin with the phenomenon. The new researcher could immerse himself or herself into the phenomenon, understand it deeply, and view it with fresh eyes, without the bias of prior theories and the interpretations of prior models and methods. Each person has a unique worldview born of his or her unique experiences and background over decades. This unique worldview is precious. It is priceless. Insight occurs when the fresh worldview of the novice meets a new phenomenon. Once the novice has fully grasped, understood, and analyzed this phenomenon, he or she is in a good position to contrast it with the current literature and challenge or refute exiting beliefs of the phenomenon.

\section{Phenomenon before theory}

Conventional wisdom advocates theory before data. The above approach espouses phenomenon before theory. This approach arises from decades of conducting research, navigating the review process, advising dozens of doctoral students and post-doctoral scholars, and steering numerous authors through the review process. Here are some reasons why this approach may have merit.

First, what is a good theory? A good theory is just a simple explanation for a phenomenon. So, without a phenomenon, there is nothing to explain. Thus, to develop a new theory, one needs careful observation of a phenomenon. Immersing oneself into the phenomenon ensures the best chance to develop a new theory.

Second, theories come and go. Phenomena stay with us forever. Phenomena may be the bedrock of theory. So, it may be better to start with the phenomenon rather than the theory.

Third, observing a phenomenon with fresh eyes unbiased by prior theory, method, or model, may enable fresh insight.

On the other hand, putting theory before data has some issues. It may lead us to borrow imperfect theories rather than develop fresh ones. It may cause researchers to complicate theories as they adapt them to the new context. It may lead to the beaten path of prior explanations rather than to new explanations. And it may result in long convoluted papers rather than short, elegant ones.

\section{How long will it take?}

Data reveal their secrets only slowly. Good research takes time. Insight takes years to crystallize. Only very tiny bits accumulate each day. Highly impactful theories take decades to formulate. Let's return to the great examples from science. 
Many people now believe in evolution. Some marvel at its beauty. However, very few know about its development. Charles Darwin, who espoused the theory, was not an evolutionist. Rather he was a naturalist, biologist, and geologist. He did not start with a grand theory of evolution. Rather he began research by observing and collecting species of plants, animals, and fossils. He also began to travel widely to better collect diverse species. He carried on such observation, collection, and reflection for about 25 years. During this time, a theory of evolution gradually began to take shape in his mind. He did so to try explain the diversity of species, their differentiation in isolated islands, their exquisite matching to various environments, and yet their common bone structures. Thus, this great theory was the fruit of a lifetime of observation, data collection, and reflection in the field. Long and careful empirics preceded and yielded great theory.

Likewise, Johannes Kepler is famous for espousing the laws of planetary motion. But how did he come to these simple laws? Kepler's mentor was Tycho Brahe. Tycho Brahe himself spent about 25 years documenting the motions of the then known six planets. He believed that the sun revolved around the earth, as was common at that time. However, Brahe had to invent numerous cycles and epicycles in each planet's motion to explain them with an earth-centered solar system. After Brahe's death, Kepler inherited these data and brought a fresh look. He began extensive analyses to try to explain Brahe's extensive data. Through the course of these analyses he arrived at various insights that led him to the formulation of three laws of planetary motion. Here again, a great theory of planetary motion emerged from decades of careful and painful empirical observation and extensive analyses of the data. In today's world with massive databases, collaborative data collection, instantaneous communication through the internet, and large communities of scientists, observation may not take a quarter century. But it does take time. And good theory will continue to emerge from fresh observation, recording, and analysis of phenomena.

\section{In praise of simplicity}

What's a good theory? A good theory is a simple explanation for a phenomenon. The best theory is the simplest explanation for a wide set of phenomena. This premise runs against many criticisms made by young reviewers. They often complain, "this theory is too simple," or "this regression model harks back to something done 20 years back." Really? So what? Simplicity is not a limitation. Simplicity is a virtue. It is the paramount virtue of a theory. Advice from theoreticians, sometimes attributed to Einstein, suggests that a theory should be as simple as possible to fully explain the phenomenon but no simpler. Now let's return to our examples from science.

Brahe's earth-centered system required numerous cycles and epicycles of planetary motion to account for the data. In contrast, with a sun-centered system and elliptical orbits, Kepler's theory required only three premises to explain the motion of planets. ${ }^{2}$ That's it. Just three premises were enough for all that data. Those three premises explained what had baffled astronomers for thousands of years from ancient Mesopotamia to medieval Europe. The theory punctured myths and religious beliefs about planetary origin and motion that had sprouted across all continents spanning millennia.

Darwin's theory of evolution is perhaps even simpler. Its original form ${ }^{3}$ required just two premises, random variation (later mutation) and natural selection. Mutations occur randomly in all species. Those that provide better adaptation to the environment get naturally selected through survival and procreation. What seemed like maddening diversity is really simple mutation and selection. What seemed purposeful is really random. What seemed divine is really natural.

\section{A plea for brevity}

Simplicity's twin virtue is brevity. If you can stay simple, you can be brief. If you strive for brevity, you will find simplicity. Actually, quality of writing improves with brevity. As we write and rewrite, we can use smaller words, use shorter sentences, trim redundant phrases, and streamline arguments. The end result is a short, forceful, idea-packed essay.

Why do our papers need to be 50 pages long? The journals with the highest impact factor in science get the job done in four pages! In that space, our papers merely cover the introduction. Have we perhaps sacrificed brevity and simplicity at the altars of complexity, jargon, and noise?

\section{Pitfalls in phenomenological approach}

The proposed phenomenological approach to create impactful and interesting research has some pitfalls. These are traps or blind spots that imperil the inferences. Here are brief notes on five of the most common of these in

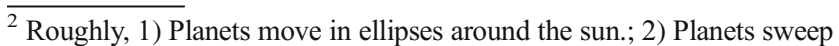
out equal areas in equal times; 3 ) The square of the planet's year divided by the cube of its mean distance from the sun is a constant.

${ }^{3}$ Developments since then have incorporated more premises to account for related phenomena.
} 
important phenomena in economics, management, and marketing.

\section{Selectivity}

Researchers typically study just a sample of the population. The danger is that the sample is selective and not representative of the population. A selective sample can lead to the wrong inference. Take pioneering advantage, for example. The sample often used to study pioneering advantage was firms that could be surveyed (Golder and Tellis 1993). However, such an approach sampled survivors and omitted failed pioneers, resulting in a severe bias in favoring of pioneering advantage. What went wrong? Researchers wanted to understand why pioneers were successful. But they sampled only surviving (successful firms). So they sampled on the dependent variable of interest. Question to ask: have I sampled on the dependent variable?

\section{Dynamics}

Most phenomena in economics, management, or marketing are dynamic. Looking at them statically loses vital dynamics that may result in wrong inferences. For example, market analysts often attribute the success of innovators to luck: being at the right place at the right time. That is surely how it seems with a one-shot look at the market when the innovator introduces the innovation that takes off. In-depth study over a long time period before the introduction would reveal the innovator's long quest, numerous missteps, lessons learned, relentless perseverance, and whole life commitment to the endeavor (Tellis and Golder 2001). Really luck had nothing to do with success. Question to ask: how does this phenomenon evolve over time?

\section{Competition}

Observers of the product lifecycle always mention decline as a necessary stage that plagues every product. Decline seems like some pre-ordained limit, just like death is encoded in an organism's genes. However, there is nothing preordained about decline. Successful products do not decline on their own due to some genetic encoding. They decline due to competition from a radical innovation that renders them obsolete (Palacios and Tellis 2015). If the firm does not embrace these innovations, competitors will. Question to ask: what impact does competition have on this phenomenon?

\section{Reverse causality}

Most models of firm behavior suggest that structure leads to strategy that leads to performance. Very few allow for a reverse path from success to strategy. However, many firms suffer from the incumbent's curse, where success leads to glorification of the past, complacency, and blindness to the next big innovation (Chandy and Tellis 2000). Success sows the seeds of failure. So, a reverse path runs from success to strategy. Ignoring such reverse causality can lead to false inferences and blindness to important insights. Question to ask: can the dependent variable affect the alleged cause?

\section{Heterogeneity}

Disruption has become a big word in today's markets. The prevailing wisdom is that established firms get disrupted by new entrants. But is this always true? Not if one considers heterogeneity in the culture of firms (Tellis 2013). A few firms realize that success sows the seeds of failure and adopt practices to avoid this trap. These firms develop an entrepreneurial culture that offers asymmetric incentives, empowers innovators, and fosters internal competition. Omitting heterogeneity in culture blinds the researcher to important insights. Question to ask: is this really equally true of all agents?

\section{You can have your cake and eat it}

The approach outlined here is tough. The path is long, lonely, and costly. Even after one collects a great deal of data, one still needs creativity. One has to see the new pattern in the whole data, as Kepler did: when the sun is set as the center of the solar system with elliptical orbits, the planets follow three neat laws. Authors need to rise above their data to have this Kepler vision or discovery. Moreover, the review process is brutal. Most papers get rejected at good journals. Many reviewers want a theory before delving into the phenomenon. Some reviewers also want consistency with the literature and may not be partial to refutation, especially if it is their own work. So positioning needs to be very careful. The toughest hurdle is criticism, which is tough to receive from others but easy to provide to others. Editors themselves may encourage critical reviews in order to make sure the paper has been properly vetted.

But if an author perseveres with the research, is immersed in the phenomenon, takes a fresh look, and sees the novel pattern, a counter-intuitive finding may emerge. Then the task falls to good framing around the surprising finding, careful positioning, simple writing, and short essays. 
Many are calling for home-grown theories. The approach outlined here allows one to have one's cake and eat it. It will allow for a home-grown theory and one that is boundary expanding. It will allow for papers that are short and interesting. It will help create research that is impactful and relevant. It will yield new phenomena and home grown theories.

We live in a time-strapped but information-profuse world. Millions of pieces of new information hit consumers every day. Who will read our work? The title of our paper needs to describe our work. But it also needs to grab an audience. It must be interesting! Being interesting is not an option. It is an imperative. It is the goal of research. The same factors that make a paper interesting will also make it impactful.

\section{References}

Chandy, R., \& Tellis, G. J. (2000). The incumbent's curse? Incumbency, size and radical product innovation. Journal of Marketing, 64(3), 1-17.

Davis, M. (1971). That's interesting! towards a phenomenology of sociology \& a sociology of phenomenology. Philosophy of the Social Sciences, 1(1971), 309-344.

Golder, P. N., \& Tellis, G. J. (1993). Pioneering advantage: marketing logic or marketing legend. Journal of Marketing Research, 158-170.

Palacios, J. \& Tellis, G. J. (2015). The dive and disruption of successful products: patterns, drivers, and predictive model. Product Innovation and Management Journal, 33(1), 53-68.

Tellis, G. J. (2013). Unrelenting innovation: How to create a culture of market dominance. San Francisco, CA: Jossey-bass.

Tellis, G. J. \& Golder, P. (2001). Will and vision: How latecomers grow to dominate markets. New York, NY: McGraw Hill. 